\title{
2. Die Romanisierung Isauriens
}

Genus hominum vagum et rapinis suetum magis quam agrorum cultibus (Sall. hist. frg. II 85). ${ }^{1}$

Gemeinhin herrscht sowohl in den Quellen als auch in der Forschung die Meinung vor, Isaurien sei zwar in den an der Küste und in der Ebene gelegenen Städten römischer Kontrolle unterworfen und zivilisiert gewesen, auf dem Lande und in den Bergen jedoch habe fortwährende Gesetzlosigkeit geherrscht, Isaurien habe sich gar in einem ständigen Aufstand befunden. ${ }^{2}$ Doch diese Ansicht kann, wie im Folgenden gezeigt werden soll, allenfalls für die zweite Hälfte des 4. Jahrhunderts und den Beginn des 5. Jahrhunderts Geltung beanspruchen, als den Versuchen der römischen Regierung, die rebellischen Bergbewohner mit militärischen Mitteln unter Kontrolle zu bekommen, zeitweise kein Erfolg beschieden war. ${ }^{3}$ Zudem wird sich im Laufe der Untersuchung zeigen, daß sich eine scharfe Trennungslinie zwischen zivilisierten und nicht zivilisierten Isauriern mit Hilfe von gegensätzlichen Kriterien wie Gebirge und Ebene angesichts des selbst in den entlegensten Ecken Isauriens zu konstatierenden hohen Urbanisierungsgrades nicht ziehen läßt.

Während der Hohen Kaiserzeit wurde die Unterwerfung und Befriedung des Taurusgebiets energisch und letztlich erfolgreich durch sich gegenseitig ergänzende Maßnahmen vorangetrieben: militärische Aktionen, Straßenbau und Urbanisierung. ${ }^{4}$ Damit besaß die Region im Gegensatz zur Piratenzeit im 1. Jahrhundert v. Chr. in den beiden folgenden Jahrhunderten einen Mischcharakter: einerseits gab es noch das Banditentum in den Bergregionen, andererseits die zivilisierte Welt in den Städten,

1 Gemeint sind die Isaurier; vgl. Maurenbrecher Sall. (1891) 94; McGushin Sall. (1992) 230 .

2 Forschungsmeinungen s.o. Einleitung; FLAM-ZuCKERMANN (1970) postuliert einen traditionellen Konflikt zwischen Stadt und Land, der sich im Banditentum im gesamten Römischen Reich manifestiert habe. Zum Stadt-Land-Gegensatz in Isaurien MitFord (1980) 1251: „It would perhaps be correct to describe the country as subject to a permanent martial law and outside its towns lawless" und SHAw (1990) passim. Die Dichotomie Berg / Ebene wird noch im Kap. III.3.2.2. im Zusammenhang mit den Erklärungen für das isaurische Banditentum diskutiert werden.

3 Magie (1950); SARTre (1991). Den radikalen Standpunkt, daß die militärischen Maßnahmen dauerhaft fehlschlugen und Isaurien tatsächlich ein quasi-autonomes Gebiet war, vertritt Hellenkemper (1986) 632f;; moderater Lenski (1999a) 421; Diskussion s.u. Kap. IV.2.3.

4 Der Romanisierungsprozeß Isauriens in der Zeit vor dem 3. Jh. ist überzeugend gegen die These des ewigen Widerstands Isauriens von SHAW (1990) dargestellt worden durch LENSKI (1999a) 431-439, dort auch umfangreiche Quellenbelege. Die Romanisierung in der frühen Kaiserzeit betont auch RovGÉ (1966) 283; zur militärischen Befriedung des Taurus Mitchell (1995a) $66 \mathrm{ff}$. 
zusätzlich ausgedehnt durch die Bewachung des Straßennetzes. Täler und Ebenen auch im Inneren des Taurusgebirges konnten sich so $\mathrm{zu}$ "Keimzellen der civilitas" entwickeln. ${ }^{5}$ Dieser Akkulturationsprozeß wäre in einer durch aggressive Widerstandshaltung aufgeladenen Stimmung in der gesamten Region nicht möglich gewesen. Nun ist der Begriff „Romanisierung“ als Bezeichnung für die Ausbreitung der römischen Lebensweise im Mittelmeerraum keineswegs unproblematisch: Handelt es sich doch beim Begriff um ein Konstrukt des 20. Jahrhunderts, beim Untersuchungsgegenstand um verschiedene Prozesse in verschiedenen Provinzen, welche auch immer von wechselseitiger Interaktion zwischen Alteingesessenen und römischen Eroberern geprägt waren. So verraten beispielsweise die Namen auf isaurischen Grabbauten eine römische Präsenz auch in den entferntesten Regionen, während ihre Ornamentik dennoch einheimisch blieb. ${ }^{6}$ Bei der Untersuchung dieser Romanisierungsprozesse vor allem für Gallien und Britannien hat sich in der neueren Forschung herauskristallisiert, daß sich die aus einer schon von den antiken Autoren postulierten Dichotomie zwischen Stadt und Land gefolgerte Auffassung vom ländlichen bzw. bergigen Raumes als Reservoir für Widerstand gegen die eindringende römische Kultur in großen Teilen als irrig erwiesen hat. $\mathrm{Zu}$ simplifizierend ist auch jener holzschnittartige Gegensatz bezüglich der Provinzialen zwischen Romanisierung und einheimischem Widerstand, wie für Isaurien von SHAW und Mitchell postuliert. ${ }^{7}$ Dennoch soll der Begriff „Romanisierung“ mutatis mutandis als Modell für den Prozeß der römischen militärischen Eroberung und den Akkulturationsprozeß der Isaurier an die griechisch-römische Zivilisation hier der Einfachheit halber verwendet werden. Dabei ist klar, daß eine vollständige Romanisierung ohnehin für keine der Provinzen festgestellt werden kann. Unter Romanisierung soll hier deshalb zunächst die Übernahme der wesentlichen munizipalen Strukturen verstanden sein.

5 LEWIN (1991) 180: „La romanizzazione aveva dunque fatto si che le valli e le pianure dell'interno divenissero dei germogli della civilitas. Il banditismo di montanari della Tracheia dovette allora fare i conti con questa nuova realtà“; ELTON (2002b) 176 betont die Rolle der Klientelkönige für die Romanisierung.

6 Mitford (1990) 2155 ff.; ER (1991) 129f.; ŞAHIN (1997) passim. Die Grabinschriften bei BM II 124-126. 127f. 141-144.

7 Problematisierung des Begriffs „Romanisierung“ und Überblick zur Debatte in den Sammelbänden von David J. MATTINGLY (Hrsg.), Dialogues in Roman Imperialism (= JRA Supplementary Series 23), Portsmouth 1997; Ray LaurenCE / Joanne BERRY (Hrsg.), Cultural Identity in the Roman Empire, London 1998, bes. Einführung 1-10; ferner bei Greg Woolf, Becoming Roman. The Origins of Provincial Civilization in Gaul, Cambridge 1998 passim, bes. 1-24 (kulturtheoretischer Überblick), 157-162 zum Konflikt zwischen Stadt und Land, 168 der Nachweis, daß es keine Lücke zwischen römischen Städten und gallischem Hinterland gegeben hat, was sich anhand der im Folgenden dargestellten Zeugnisse auf Isaurien übertragen läßt; knapp bei LENSKI (1999a) 434. 


\subsection{Die Militärverwaltung Isauriens während der Hohen Kaiserzeit}

In augusteischer Zeit, als Isaurien teils zur Provinz Galatia, teils zum Klientelkönigreich des Polemo und seines Nachfolgers Archelaus gehörte, diente das pisidische Antiochia der legio VII Macedonica als Ausgangsbasis für Operationen im Taurusgebirge, falls den mit Rom verbündeten Dynasten gegen aufständische Stämme geholfen werden mußte. ${ }^{8}$ Das Schicksal des Galaterkönigs Amyntas, der im Kampf gegen die aufständischen Homonadeis umgekommen war, ${ }^{9}$ sollte sich nicht wiederholen. Hinzu kamen zwei Kohorten und drei alae in der südlichen Hälfte der Galatia. Die pisidischen Kolonien ${ }^{10}$ hatten somit die Funktion, das noch nicht provinzialisierte Isaurien militärisch in Schach zu halten bzw. das Einsickern von Banditen, wie es im Verlauf des 3. Jahrhunderts dann erfolgte, zu verhindern. Die Militärpräsenz in Isaurien konnte nach der augusteischen Zeit verringert werden und nahm erst wieder Ende des 3. Jahrhunderts infolge der Unruhen zu. ${ }^{11}$

Die Romanisierung des Taurusgebiets wurde unter der julisch-claudischen Dynastie durch Städtegründungen stark vorangetrieben. Dies zeigen die Namen der Neugründungen: Tiberius hatte Germanicopolis gegründet, und Claudius drückte mit Hilfe seines umtriebigen Legaten M. Annius Afrinus (49-54 n. Chr.) der Galatia mit den Gründungen Claudiopolis, Claudioderbe, Claudiconium, Claudiolaodicea, Claudiocaesarea Mistea und Claudioseleucia seinen Stempel auf. Afrinus ließ eine Inschrift für Claudius am isaurischen Trogitis-See errichten, und sein Name und Porträt tauchen auf Münzen aus Iconium und Pessinus auf. ${ }^{12}$ Der durch Verhandlungsgeschick gelöste Konflikt mit den Kieten im Jahr 52 beendete den verbliebenen einheimischen Widerstand. ${ }^{13}$ Bereits unter römischer Kontrolle stehendes Territorium wurde durch Koloniegründungen gesichert, die Kommunikationslinien wurden durch den Bau der via Sebaste verbessert. Man begann rasch, auch die Isaurier selbst für die römischen Legionen zu rekrutieren; so waren isaurische bzw. homonadensische Rekruten aus Balat im Bergland westlich des Trogitis-Sees Legionäre der VI Ferrata. ${ }^{14}$

Rom war schließlich überall in der Region mit zahlreichen Veteranenkolonien engagiert. Von deren Anlage versprach man sich einen doppelten Nutzeffekt: Zum einen waren die Veteranen mit Land versorgt, zum anderen konnte dieses romanisiert und unter Kontrolle gehalten werden. Ein Veteran aus der Flotte von Misenum ließ sich in Selinus-Traianopolis bestatten. ${ }^{15}$ Auch in der Polis Isaura, die fern von der

8 Erich S. Gruen, $C A H X\left({ }^{2} 1996\right) 151-154$ u. o. Kap. II.1.1.

9 S.o. Kap. III.1.6.

10 Mon. Anc. 28; vgl. Mitchell (1995a) 73-79.

11 IGR III 284 in Isaura Palaia; vgl. MitCheLL (1995a) 122 u. Anm. 36.

12 CIL III 6799; PIR ${ }^{2}$ A 630; RamSAy (1922) 159; MitChell (1995a) 66f. 78f. u. Anm. 94 ff.

13 Tac. ann. XII 55; s.o. Kap. III.1.6.

14 BM II 21, Nr. 4; MitCHELl (1995a) 140.

15 Repertorium 378, 'SIT 2' = CIL III 1, 225; CIL III Suppl. 1, p. 1230. 
Küste und bedeutsamen Verkehrsstraßen gelegen war, waren seit der julischclaudischen Zeit Römer ansässig. Sie formten als Veteranen innerhalb der Verwaltungsstruktur in Kooperation mit der Bovג regrine Rätegemeinde innerhalb der sonst selbständigen civitas. ${ }^{16}$ Isaura war nicht nur Sitz von Veteranen, sondern auch Rekrutierungsraum: Soldaten der legio XVI Flavia Firma und der $X V$ Apollinaris sind aus dem Territorium der Stadt eingezogen worden. ${ }^{17}$ In mehreren weiteren Orten Isauriens sind inschriftlich römische Legionäre bezeugt. ${ }^{18}$ Eine zweite Stufe im Romanisierungsprozeß erfolgte mit der Einrichtung der neuen Provinz durch Vespasian, was an der hohen Anzahl der Flavius-Namen bei den Legionären, die sich auch in Isaura Nova ansiedelten, abzulesen ist. ${ }^{19}$ Isaura bekam schließlich den Status einer reinen Bürgergemeinde, einer colonia. ${ }^{20}$

Vespasian drängte den zuvor starken Einfluß lokaler Potentaten zurück: Die priesterliche Teukridendynastie in Olba war spätestens mit Einrichtung der Provinz Cilicia durch diesen Kaiser entmachtet. Bereits zu augusteischer Zeit, unmittelbar nach der Zerschlagung des Zeus-Olbios-Kultes als dynastischer Legitimation für die Priesterdynastie, hatte der Klientelkönig Polemo auf den Titel des Erzpriesters ( $a r$ chiereus) verzichtet. Der Romanisierungsverlauf in Kleinasien läßt sich so am Beispiel der olbischen Priesterdynastie zeigen: Die großen Poleis Olba und Diocaesarea erwuchsen aus einem Klientelfürstentum, es wurde so eine Landschaft von der Peripherie her romanisiert. $^{2 !}$

\subsection{Straßen}

Die wichtigste Voraussetzung für die Romanisierung war eine funktionierende Infrastruktur: Rom baute seit der Eingliederung Isauriens in die Provinz Cilicia im Jahr 63 v. Chr. Straßen durch das gesamte isaurische Hinterland und sicherte diese durch

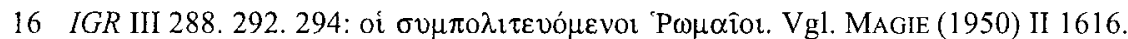

$17 W E$ Nr. $27=I G R$ III 281 (aus Yali Hüyük, einem Dorf direkt unterhalb von Isaura Nova); vgl. Michael P. SPEIDEL, 'Legionary Recruiting from Asia Minor' in: $A N R W$ II 7 (1980), 730-746; MitCheLl (1995a) 136. 139f. u. Anm. 179. Dagegen dürften die drei isaurischen Legionen, ND or. VII 56, gerade nicht aus Einheimischen rekrutiert worden sein, wie HopwOOD (1986) 345 annimmt.

18 Aufgelistet mit Angabe der Editionen bei Laminger-PASCHER (1985) 387-391; vgl. RaMSAY (1941) 159. 217f. 224. 228; HoPWOOD (1986) 345.

19 CIL III 6785: T. Flavio Castoris / P. Cyr. Alexandro / civitate donato ab / Imp. Caes. Vespasiano / P. Hermes lib. Vgl. Ramsay (1941) 202: „The sudden appearance of Flavian cives in the Isaurikan region indicates an attempt made by Vespasian to turn the Isauri and neighbouring provincials into loyal supporters of the Empire. His method was the usual one, viz. to reward the philo-Roman and to neglect the recalcitrant, and to trust to time."

20 Anonymus Ravennatis II 17, 14 p. 30 : Colonia Isauria.

21 GotTer (2001) 303, 314. 
Patrouillen und Streckenposten. ${ }^{22}$ Diese Streckenposten waren nicht unbedingt aufgrund einer akuten Gefährdung einer Strecke geschaffen worden, zunächst waren sie für die ständige Überwachung und Sicherung des cursus publicus vorgesehen. ${ }^{23}$ Selbst im krisengeschüttelten 3. Jahrhundert, als das Straßennetz aus staatlichem Geldmangel vielerorts vernachlässigt wurde, hielt man die Straße von Iconium nach Isaurien in Ordnung. ${ }^{24}$

Die im Jahr 6 v. Chr. von Augustus als Fahrstraße gebaute via Sebaste verlief durch Pamphylien und Pisidien von Side aus nördlich des Taurusmassivs über das pisidische Antiochia nach Iconium; der von Antiochia aus gezählte 140. Meilenstein steht noch immer in situ auf der Paßhöhe bei Comama. ${ }^{25}$ Paulus und Barnabas gelangten hier auf ihrer Missionsreise von Perge ins pisidische Antiochia. ${ }^{26}$ Eine weitere Straße, auf der vermutlich bereits P. Servilius während seiner Kampagne im 1. Jahrhundert v. Chr. gegen die Isaurier marschiert war, verlief durch das Cestrus- (Aksu-) Tal an Kremna vorbei nach Sagalassus, eine weitere verlief nordöstlich von Side am Melas entlang, durch die Berge zum Bassin des Beyşehir- (Caralis-) Sees, von dort durch die lykaonische Ebene nach Iconium in Lykaonien. ${ }^{27}$ Unter Vespasian wurden die Straßen im gesamten Provinzkomplex Galatia et Cappadocia erneuert. ${ }^{28}$ Eine wohl unter Hadrian befestigte Straße verband die Küste von Anemurium aus mit dem Inland, sie verlief über Titiopolis über Eirenopolis nach Germanicopolis und von dort aus über einen Höhenzug nach Antiochia und Laranda. ${ }^{29}$ Eine weitere verband Iconium über Laranda mit Seleucia an der Küste. ${ }^{30}$ Mit diesem Straßennetz war eine weit-

22 BM II 210. 211 (Meilensteine); TIB 5,1 128-140; Ballance (1958); French (1974). (1980). (1988); Hopwood (1991); Mitchell (1995a) 77, Anm. 78; Sartre (1991) 79f.; SAHIN (1997) 75, Anm. 5 (Forschungsüberblick); ElTON (2002b) 181f.

23 William H. C. FREND, "A third Century Inscription relating to Angareia in Phrygia' in: JRS 46 (1956), 46-56; Ramsay MACMulLeN, Soldier and Civilian in the Later Roman Empire, Cambridge, Mass. 1963, 55-59; LEWIN (1991) 174. 182, Anm. 33.

24 CIL III $6956=12215$; Meilenstein gefunden in Kavak auf der Route Iconium-Isauria; MAGIE (1950) I 719. II 1572, Anm. 36. 1576, Anm. 48.

25 Levick (1967) 39; Liste der Meilensteine bei French (1980) 708, 727; Mitchell (1995a) 70.

26 Apg. 13, 9.

27 Mitchell (1995a) 70; Servilius marschierte eher durch das Cestrus- als durch das Melastal, vgl. KeySER (1997) 74 u. Anm. 49 u. o. Kap. II.1.3.

28 ILS 263. 268.

29 SCHAFFER (1903) nahm einen direkten Straßenverlauf an; BM II 189. 206 einen gekrümmten. Es wurden Meilensteine und Reste einer mansio gefunden, vgl. BM II 189 ff.; HopWOOD (1991) 306. MITFORD (1980) 1248 schließt aus BM II 189 Nr. 210 auf einen Bau dieser Straße unter Hadrian.

30 Amm. XIV 25; RAMSAY (1890) 361; MACKay/ MACKaY (1969); SAYAR (1992). 
gehende Kontrolle und die schnelle Truppenverlegung in Unruhegebiete möglich. ${ }^{31}$ Das Postwesen blieb trotz der großen isaurischen Aufstände intakt: in den Miracula der Heiligen Thekla ist ein Meldereiter erwähnt. ${ }^{32}$

Die Streckenposten (stationes) sollten den Frieden auf den Straßen garantieren. Sie wurden zumeist von einem stationarius oder beneficiarius geleitet. Dieser war ein Soldat, der seine Beförderung einem beneficium seines Vorgesetzten verdankte und auch rechtliche und finanzielle Funktionen ausübte. In Isaurien ist ein stationarius in Gorgorome (Akkise) auf dem Territorium der Homonadeis am Trogitis-See bezeugt, ein weiterer in Artanada südwestlich von Isaura. ${ }^{33}$ Auch Isaurier waren als stationarii in den Nachbarprovinzen tätig, so ist ein Isaurier namens Aelius Telephus auf dem Olymp an der felsigen lykischen Südostküste durch eine von seiner Frau auf der statio gesetzte Inschrift in diesem Amt bezeugt. ${ }^{34}$ Auf der ebenfalls durch einen stationarius gesicherten Küstenstraße von Laertes (Çebel Ires) in Ost-Pamphylien nach Syedra in Isaurien patrouillierte unter Hadrian und Antoninus Pius eine Militäreinheit und sicherte so die enge Küstenlinie südlich des Taurusmassivs. ${ }^{35}$ Die Verbindungen der

31 Hellenkemper (1986) 628 geht mit seiner Folgerung, wonach die Stationierung von Truppen in Isaurien und in den angrenzenden Provinzen wegen der Unpassierbarkeit der Straßen aufgrund der winterlichen Schneefälle keinerlei Abschreckung auf die Taurusstämme geboten haben soll, wohl zu weit; in diesem Sinne aber auch Terence B. MiTFORD, 'High and Low Level Routes across the Taurus and Antitaurus' in: FrenCH / LightFoOT (1989) 329-333, bezogen auf den kappadokischen Limes gegen die Sasaniden. Noch 1963 war diese Gegend im Winter ebenso unpassierbar wie in der Antike. Hierbei ist allerdings zu bedenken, daß die hochgelegenen Paßstraßen in diesem Fall nicht nur für die Legionäre, sondern auch für die Banditen unpassierbar waren. Noch heute ist die Straße von Isaura Vetus (Bozkır) durch die isaurische Dekapolis nach Germanicopolis (Ermenek) durch die Taurusschluchten teilweise unbefestigt.

32 Mir. 16; TIB 5,1 129.

33 Gorgorome: IGR III 280; Hall (1971), 129 ff.; $A E$ (1972) 660f. Artanada: IGR III 812; RUSSELL (1991) 487. Zu den stationarii allgemein: Friedrich LAMMERT, s.v. 'stationarii', RE III A 2 (1929) 2213; Robert Hellenica XIII (1965) 80; Syme (1987) 145. Eine Definition des beneficiarius gibt Veg. mil. II 7; vgl. Yann Le BOHEC, s. v. 'Beneficiarii', DNP 2 (1997), 561; Alfred v. DoMASZEWSKI, 'Die Beneficiarposten und die römischen Straßennetze' in: Westdeutsche Zeitschrift für Geschichte und Kunst 21 (1902) 158-211; THOMPSON (1947) 130; MacMulden (1966) 260; Sünskes Thompson (1990) 200; Joachim Ott, Die Beneficiarier. Untersuchungen zu ihrer Stellung innerhalb der Rangordnung des römischen Heeres und zu ihrer Funktion (= Historia Einzelschriften 92), Stuttgart 1995, erwähnt in seinem Corpus der die Beneficiarier betreffenden Inschriften die obengenannten nicht; vgl. die Rez. von Boris Rankow in CR n.s. 49 (1999), $182 f$.

34 IGR III $748=$ TAM II 3, 1165 sowie 953.957 (zwei weitere stationarii auf dem lykischen Olymp); RoBert Hellenica X (1955) 172-177; Mitchell (1995a) 122.

35 RusSELL (1991) nach einem Militärdiplom, die Identifikation des Ortes 484 ff., ebenso BM II 97-105; BRANDT (1992) 9; Mitchell (1995a) 122. 
statio zu den umliegenden Gemeinden beschleunigten die Vermischung der dort über mehrere Jahre ansässigen Besatzungen mit der einheimischen Bevölkerung. ${ }^{36}$

\subsection{Urbanisierung}

Die hellenistische und römische Kultur war nicht nur in den Städten an der Küste und in der Ebene um Claudiopolis, ${ }^{37}$ sondern auch in den hochgelegenen isaurischen „Hinterland"-Poleis präsent. ${ }^{38}$ Die Urbanisation isaurischer Siedlungen und die Kooperation der lokalen mit den römischen Autoritäten spätestens seit der Befriedung Kilikiens durch Pompeius nach der Schlacht von Corasium kann angesichts der numismatischen und epigraphischen Evidenz nicht geleugnet werden.$^{39}$ Als ein repräsentatives Beispiel mögen der in zwei Inschriften aus dem 2. Jahrhundert bezeugte lokale Potentat Stanamoas und seine Familie aus Cotenna am Melas, dem Isaurien westlich begrenzenden Fluß, angeführt werden. Aus den Inschriften geht hervor, daß Stanamoas, ebenso wie sein Bruder und Sohn mehrmals Priester des Kaiserkultes, Archon und Eirenarch gewesen ist; seine Tochter und Schwiegertochter waren Artemispriesterinnen. Zweimal unternahm er Gesandtschaftsreisen nach Rom. ${ }^{40}$ Von dem seit der Provinzialisierung Isauriens durch Vespasian funktionierenden Kaiserkult zeugen die Ehrenbögen in Isaura Nova mit Weihinschriften für Hadrian, Marc Aurel und Septimius Severus. ${ }^{41}$

36 Vgl. RusSELL (1991) 488.

37 Strab. XIV 4, 3-7; EYICE (1981) passim.

38 Jones (21971) 137 ff.; RigHINI (1976); HoPwOod (1983); SHAw (1990) 232 kritisiert zwar, daß alle Beispiele Hopwoods für die Romanisierung aus der Ebene stammten. Doch es finden sich auch Beispiele aus dem Hochland, allen voran Isaura Nova, vgl. LENSKI (1999a) 433. Die Tatsache des hohen Urbanisierungsgrades gerade in diesen Gegenden relativiert somit auch den Begriff „Hinterland“, mit dem v.a. HopwOoD in seinen Arbeiten operiert. LENSKI (1999a) 431, Anm. 92 definiert das isaurische Hinterland als die bergige Gegend südlich der lykaonischen Ebene ab einer Entfernung von der Küste von $15 \mathrm{~km}$.

39 So schon BENT (1891) 206; LENSKI (1999a) 432 ff. mit umfassendem Überblick über die Quellen.

40 Repertorium 95 ff., 'Göd 1-2' = BÉ (1972) 496; 'Göd 3' = $A \dot{E}$ (1972) 625; BM II 30-33, Nr. 12f.; Hopwood (1984) passim; zum Kult Jones ( $\left.{ }^{2} 1971\right) 140$.

$41 W E$ Nr. 180, 181, 184, 193; IGR III 280 (Gorgoromene am Trogitissee). 292. 293 (Isaura Nova); MAMA III Nr. 152; RAMSAY (1941) 202: "It was probably under the Flavians that a high priesthood of the Emperors was created at Palaia [id est: Nea, K.F.] Isaura. We find no case of a Claudian civis region." Katalog der Inschriften zum Kaiserkult bei MiTforD (1990) 2152-2155; ELTON (2002b) 180. Freilich kann aus der alleinigen Tatsache eines Kaiserkults nicht schon auf einen hohen Romanisierungsgrad geschlossen werden, gibt es doch auch Fälle von Kaiserkult außerhalb des Reiches, z.B. Suet. Augustus 60. Dies ist jedoch ein Beleg für die Attraktivität des Imperium Romanum auf seine Randgebiete und verdeutlicht den gerade von Nichtrömern getragenen Romanisierungsprozeß. 
Die Stadt war somit mitnichten das Räubernest, als das sie aufgrund des Eindrucks aus den schriftlichen Quellen von ihren Besuchern HAMILTON, DAVIS und VERZONE gesehen wurde, oder der Sitz einer durch vorkulturelle Sozialformen geprägten, dem römischen System völlig entgegengesetzten Gesellschaft, worin selbst die Eliten völlig immun gegen jede Form der Akkulturation sind, wie von SHAw dargestellt. ${ }^{42}$ Ein Einwohner Isauras ließ unter Marc Aurel eine Stoa mit 25 Säulen und dazwischenliegenden Läden errichten. ${ }^{43}$ Aus Isaurien stammende Römer waren stolz auf ihre Heimatstadt: eine von dort stammende Frau setzte ihrem Mann im lykaonischen Lystra eine Ehreninschrift, auf der sie seine Herkunft betonte. ${ }^{44}$ Die Münzprägung von Isaura Nova und anderen isaurischen Poleis bezeugt deren Polis-, bzw. im Falle Isauras, ihren Metropolis-Rang. ${ }^{45}$ Neben Isaura Nova läßt sich für fünf weitere Städte des „Hinterlands“ eine Boule nachweisen: Amblada (Yeniçe), Artanada (Dülgerler), Astra (Tamaşalik) und Coropissus. ${ }^{46}$ Zwar läßt sich auf dem Territorium von Isaura selbst kein Theater finden, doch im (heute überbauten) Claudiopolis gibt es Hinweise auf die Überreste eines solchen. ${ }^{47}$ Die Urbanisierung zeigt sich auch in Sportanlagen: In drei isaurischen Städten lassen sich Stadien nachweisen, so in Seleucia, Lamus sowie im landeinwärts in bergiger Gegend gelegenen Coropissus bei Claudiopolis. Die Romanisierung Isauriens schlug sich letztlich im Bildungsgrad seiner Bewohner nieder: In der Spätantike ist eine Reihe von aus Isaurien stammenden Gelehrten bekannt. ${ }^{48}$ Die Isaurier wurden während der Hohen Kaiserzeit nicht mehr als grundsätzlich Fremde angesehen - wäre dies der Fall gewesen, dann gäbe es auch nicht die zahlreichen Quellenaussagen über ihre plötzliche Re-Barbarisierung. ${ }^{49}$

Insgesamt verfügte das Rauhe Kilikien trotz seiner Zerklüftung über einen beachtlich hohen Urbanisierungsgrad. Gerade im bergigen Binnenland gab es eine hohe

42 SHAw (1990) 261; dazu auch Mitchell (1995a) 77, Anm. 74: „The important general study by SHAW [...] neglects to consider the specific ways in which the Highlands were controlled under the Romans".

43 IGR III 287; Denkmäler 147; ROBERT (1987) 486.

44 Laminger-PASCHER ETAM 15 (1992) 127, Nr. $168=$ MAMA VIII p. 3, Nr. 12 mit Taf. 2.

45 CGC 21, XXVII. 13F.; SNG 5408-5412. 5762--5781; LENSKI (1999a) 432.

46 Amblada: Hall (1968) Nr. 21; Artanada: WE Nr. 96, vgl. TIB 5,1 198 s.v. 'Artanada'; Astra, $7 \mathrm{~km}$ nördlich von Hadim am Oberlauf des Kalykadnos gelegen: BM II 129-136 Nr. 121, vgl. TIB 5,1 200f. s.v. 'Astra'; Coropissus: OGIS 574, Philadelphia: BM II Nr. 246; vgl. LENSKI (1999a) 432 mit Anm. 95.

47 Denkmäler 125-136.

48 S.u. Kap. V.1.3.

49 Zur Rebarbarisierung s.u. IV.3.3. Zur Einschätzung der Nichtrömer in der lateinischen Literatur während des aufstrebenden Imperiums vgl. John P.V.D. BALSDON, Romans and Aliens, London 1979. 
Anzahl von Städten, und dies in einem weitaus höheren Maße als heute. ${ }^{50}$ Die Anzahl der Städte dort war zur Zeit des Hierokles um 530 sogar höher als derjenigen im Flachen Kilikien, allein im südlichen, nicht zu Lykaonien gehörigen Isaurien stehen 23 Städte gegen 17 im Flachen Kilikien. ${ }^{51}$ Das Verhältnis zwischen Stadt und Land oder, wenn man so will, Ebene und Berg war infolgedessen keineswegs zerrüttet, sondern beide Teile ergänzten sich und waren aufeinander angewiesen, worauf im Folgenden noch genauer eingegangen wird.

\subsection{Die Verflechtung des ländlichen Raumes mit den Städten}

Im Zusammenhang mit der Erosion staatlicher Zentralgewalt in der Spätantike wirft sich für Isaurien die Frage auf, ob es im römischen Reich insgesamt, in Kleinasien oder doch zumindest in Isaurien ein Gefälle zwischen Stadt und Land gegeben hat, bzw. ob gar der ländliche Raum in der Spätantike überhaupt als ein quasi rechtsfreier Raum anzusehen ist, und ob dieser Sachverhalt gerade in Isaurien durch die außerordentlich hohe Unzugänglichkeit seines ländlichen Raumes noch verstärkt wurde, ${ }^{52}$ oder ob angesichts der in dieser Arbeit schon vielfach festgestellten gegenseitigen Abhängigkeiten zwischen Stadt und Land dieser Gegensatz zumindest nicht unmittelbar entscheidend für das räuberische Verhalten der Isaurier gewesen sein kann. ${ }^{53}$

Der innere Zusammenhang zwischen vorgegebenem Naturraum und menschlichem Verhalten ist ein Topos der antiken Historiographie. Die bekannteste derartige Schrift dürfte „Über die Umwelt" aus dem Corpus Hippocraticum sein. Poseidonius (135-50 v. Chr.) entwickelte eine regelrechte „Klimatheorie“, wonach sich das Klima auf den Charakter der Bewohner in den jeweiligen Landschaften ausgewirkt haben soll. ${ }^{54}$ Im Falle Isauriens wird die rauhe Bergwelt mit einer Affinität ihrer Bewohner zum Banditentum in Entsprechung gesehen. So unterscheidet Strabo die ,guten“

$50 \mathrm{Vgl}$. die Karten in TIB 5,1; zur Urbanisierung TOMASChitz (1998) 37. Aus diesem Grunde kann auch eine „Ferne“ des urbanen Lebens in Anatolien, die SARTRE (1995) 209 postuliert, nicht einmal für eine so abgelegene Gegend wie Isaurien als ganzes gelten.

51 Hierocl. 708 ohne 672 im Vergleich zu 704f.; vgl. LENSKI (1999a) 433 mit Anm. 98.

52 So beispielsweise bei Shaw (1984). (1990); Mitchell (1995a) 197; Sartre (1995) 271.

$53 \mathrm{Zu}$ diesem Ergebnis für Lykien und Pamphylien kommt BRANDT (1992).

54 Hippocrates, De aere aquis locis, ed. u. dt. Übers. Hans Diller, (Corpus Medicorum Graecorum I 1, 2), Berlin 1970; Jürgen Malitz, Die Historien des Poseidonius, München 1983, 81-85; Allan A. LuND, Zum Germanenbild der Römer. Eine Einführung in die antike Ethnographie, Heidelberg 1990, 35-55; David LENFANT, 'Milieu naturel et différences ethniques dans la pensée grecque classique' in: Ktéma 16 (1991), 111-122; Benjamin H. ISAAC, The Invention of Racism in Classical Antiquity, Princeton 2004, bes. 55-168. 
Kilikier der Ebene von den „schlechten“ des Berglands, die dann stellvertretend für alle Piraten des östlichen Mittelmeerraumes standen. ${ }^{55}$

Nach Strabo konnten unterschiedliche geographische und historische Einflüsse sogar ein und dasselbe Volk zu verschiedenen Charakteren formen; für diese These boten die Kilikier das Paradebeispiel. Strabo ersetzte die alte Dichotomie zwischen unzivilisierten Inland- und zivilisierten Küstenbewohnern aus der Zeit der griechischen Kolonisation durch jene zwischen Berg- und Talbewohnern und wandte damit ein Schema an, in welchem er die Völker zwischen den Polen Zivilisation und Barbarentum einordnete. ${ }^{56}$ Dabei wurden deren Eigenschaften zunächst beschrieben, dann wurden die Völker einer Bewertung unterzogen. Seine historische Analyse diente der Erklärung der zivilisatorischen Errungenschaften jedes Volkes, vor allem der Glorifizierung der Verdienste Roms. ${ }^{57}$

Doch nicht alle antiken Autoren übernahmen die Ansicht, daß die Landschaft den Charakter der Bewohner präge. So spricht der Autor der Historia Augusta polemisierend den Isauriern jegliche Kriegstauglichkeit ab. Die Gefährlichkeit der isaurischen Banditen sei allein der Tatsache geschuldet, daß sie sich immer in das unzugängliche Gebirge zurückziehen könnten. Deshalb hätten es die ,guten“ Senatskaisern seit dem 3. Jahrhundert öfters geplant, so seine wiederum wohl erfundene Behauptung, sie in die Ebene umzusiedeln. ${ }^{58}$

55 Paolo Desideri / Salvatore Settis: 'La Cilicia e i Cilici fra montagna e pianura' in: Premessa zu [der Kilikien gewidmeten Ausgabe der] Quaderni Strorici 76 (1991), 7 ff.

56 DESIDERI (1991a) $300 \mathrm{zu}$ Strab. II 5, 32. XIV 5,1.

57 Thollard (1987) 84f.; vgl. auch François LasSERRE, 'Strabon devant l'empire Romain' in: $A N R W$ II 30, 1 (1982), 867-896. 889. 893 ff.: Strabo nahm in seinem Werk auf das Imperium eher eine Außen- als eine Innensicht ein. Dabei hat er als Grieche zwar Ressentiments gegen die römische Okkupation, versucht jedoch, Lokalpatriotismus und Bewunderung für die Garantie von Freiheit und Ordnung durch das Imperium in Einklang zu bringen. GARNSEY (1988) 204f. argumentiert gegen die deterministische Sicht Strabos von den Bergen als vegetationsarmer Gegend, welche ihre Bewohner zu Banditen mache. Es gebe mehr politische als geographische Gründe für die Transhumanz; die baumarme Graslandschaft sei in der Regel menschengemacht. Auch BRANDT (1992) 22 polemisiert gegen die (Strabo folgende) Sichtweise von Helmut WEIMERT, Wirtschaft als landschaftsgebundenes Phänomen. Die antike Landschaft Pontos. Eine Fallstudie, Frankfurt a. M. 1984, 7f.: die These, daß allein naturräumliche Bedingungen die wirtschaftlichen Verhältnisse in einem Gebiet prägten und nicht etwa politische Umstände, sei irrig. Nach EQUINI SCHNEIDER (1999) 27-33. 28 hat auch Ptol. Geogr. V 5, 3. 5, 8 die problematische strabonische Zweiteilung in rauhes und flaches Kilikien übernommen. Zu Strabos Klassifizierung der ökonomischen Prosperität vgl. den Artikel von Daniele Foraboschi in Biraschi / SalmeRI (2000).

58 SHA tyr. trig. 26, 7: Nam sunt non statura decori, non virtute graves, non instructi armis, non consiliis prudentes, sed hoc solo securi, quod in editis positi adiri nequeunt. Quos quidem divus Claudius paene ad hoc perduxerat, ut a suis semotos locis in Cilicia conlocaret. Zur historischen Glaubwürdigkeit vgl. III.1.2. 
Die Gleichsetzung von Landschaftsprofil und Charakter war also bereits in der Antike nicht allgemein anerkannt. Überhaupt sind Zweifel angebracht, ob die Interpretation Strabos der historischen Realität Genüge tut: Wie schon oben dargelegt, war der Urbanisierungsgrad in Isaurien weit fortgeschritten. Dennoch ist die vorgegebene Dichotomie zwischen Stadt und Land auch für die moderne Forschung verführerisch: Nach Stephen MiTCHELL hat sich der „kulturelle Graben“ zwischen Stadt und Land in Kleinasien gerade in der Spätantike durch die rigidere Steuergesetzgebung vergrößert. Die städtischen Magistrate hatten in verstärktem Maße für das Steueraufkommen der Landbevölkerung zu sorgen. So ernannten die Magistrate der Polis Aphrodisias jähr-

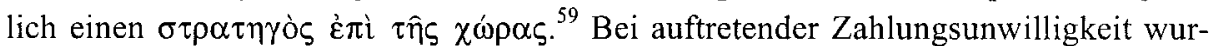
den Hilfspolizisten, sogenannte Diogmiten ${ }^{60}$, als Rollkommandos eingesetzt. So sei zu dieser Zeit eine neue Dynamik in die Beziehungen zwischen Stadt und Land gekommen, welche die Entfremdung noch verstärkt habe, vor allem, wenn die besteuerte Bevölkerung aus Hirten bestand, die bei einem Aufstand wenig zu verlieren hatten und deren innere Schwelle zur gewaltsamen Erhebung niedriger war. ${ }^{61}$ Besonders in Isaurien sei das Wohlstandsgefälle zwischen Stadt und Land sehr groß gewesen, da es außerhalb der kleinen Poleis keine wohlhabenden Gutsbesitzer gegeben habe - Stadt und Land seien somit ,getrennte Welten“ gewesen. ${ }^{62}$

Dagegen lassen sich die folgenden Überlegungen anführen: ${ }^{63}$ Angesichts der für die zerklüfteten geographischen Verhältnisse recht hohen Siedlungsdichte ist es generell fraglich, wie und wo in Isaurien die Grenze zwischen Stadt und ,,ändlichem

59 Mitchell (1995a) 195.

60 S.u. IV.3.1.2.

61 HoPWOOD (1983) 173.

62 Mitchell (1995a) 195, 197 zum Verhältnis von Stadt und Land in Kleinasien als ,worlds apart" zwischen hellenisierten Polisbewohnern und indigenen Landbewohnern, und dem Verweis, daß die Landbewohner selbst für den die Rechtssicherheit und den Frieden im Imperium Romanum der Hohen Kaiserzeit preisenden Aelius Aristides eine ständige Be-

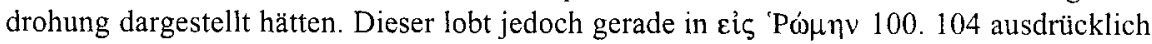
die gefahrlose Reisefreiheit. MrTCHELL (1995a) 197, gefolgt von RIESs (2001) 52, interpretiert zudem die oben beschriebene Rolle der ländlichen Hilfspolizei wie folgt: „The oppressive role of the rural gendarmerie is the most conspicuous symptom of a general condition, that hellenized city dwellers and native villagers lived in worlds apart. The terminology of Roman inscriptions which tend to suppress or marginalize the formal distinctions between urban and rural inhabitants may suggest otherwise, but the remainder of the evidence confirms that the divide between the citizens and the perioikoi of the Hellenistic period remained as pronounced as ever."

63 Vgl. auch SCHULER (1998) 273-288, 287f. gegen Mitchell mit dem Verweis das anatolische Binnenland, auch in Bezug auf bergige Gegenden wie Isaurien. Die Terminologie der kommunalen Institutionen der Landgemeinden unterscheide sich wenig von der Inschriftensprache der Poleis und bestätige gerade die Vertrautheit „zumindest eines Teils der Dorfbewohner mit dem städtischen Geschehen“; ebenso LENSKI (1999a) 417. 
Raum", mithin zwischen bewohntem, begehbarem und bewirtschaftetem Raum, gezogen werden soll. Dabei ist vor allem zu bedenken, daß für die extensive Weidewirtschaft, die in Isaurien vorrangig betrieben wurde, andere Größenordnungen als für intensive Landwirtschaft in anderen Gegenden gelten. Insofern ist die Trennung zwischen Stadt und Land im zerklüfteten Isaurien von anderer Qualität als in ebenen Landstrichen: Der ländliche Raum wurde bis in große Höhen dauerhaft besiedelt und bewirtschaftet. Selbst an Steilhängen wurde im Sommer Weidewirtschaft betrieben und der Boden durch Terrassierung agrarisch nutzbar gemacht. Noch im Mittelalter war der ländliche Raum in Isaurien überproportional dicht besiedelt, ${ }^{64}$ auch durch zahlreiche Einzelgehöfte und Weiler in Streulage, wenngleich diese Funde nicht durch systematische Untersuchungen gedeckt sind. ${ }^{65}$ Einer dieser Weiler, Gökburç, $14 \mathrm{~km}$ nördlich von Seleucia an einer Straßengabelung gelegen, existierte schon in hellenistischer Zeit. Die relativ hohe Siedlungsdichte selbst in den bergigen Regionen zeigt also, daß dort ausreichende Lebensgrundlagen vorhanden waren. ${ }^{66}$ Erst im 4. Jahrhundert wurde es erneut befestigt und dokumentiert das Schwinden der staatlichen Autorität, denn die Befestigung von Gehöften war erst ab 420 nolens volens gesetzlich gestattet. ${ }^{67}$

Auf dem Land stieg der Wohlstand in dieser Zeit, was nicht auf eine Ausbeutung des Umlandes durch die Städter schließen läßt. ${ }^{68}$ Die Beziehungen zwischen den Städten und dem ländlichen Raum, von den Raubzügen abgesehen, scheinen sehr gut gewesen zu sein: Im Stadion von Seleucia besaß eine Dorfgemeinde eigene Plätze. ${ }^{69}$ Die von Ammian beschriebene Vermittlertätigkeit der Einwohner von Germanicopolis beim Aufstand von 368 gibt einen Hinweis auf die enge Verbindung zwischen Stadt- und Landbewohnern. ${ }^{70}$ Die ländlichen Gebiete waren selbst im Hochland schon frühzeitig bevölkerungsreich, dies zeigt die Nachricht von den 4000 Kriegsgefangenen, welche die Homonadeis im pisidischen Hochland im Krieg gegen Amyntas und die Römer verloren. ${ }^{71}$

Nach Hansgerd Hellenkemper hat sich die Stadtbevölkerung weder ethnisch noch sprachlich den Isauriern verbunden gefühlt. Er führt zum Beleg eine Stelle in den

64 EYICE (1981) 207f. mit Taf. 81-89.

65 SCHULER (1998) 84.

66 HellenkemPer (1986) 626 gegen die These von Mango (1966) 363, die Isaurier hätten wenig Landwirtschaft betrieben und seien deshalb auf die Steinmetzkunst angewiesen gewesen, die sie dann exportierten, mit dem Hinweis auf die Differenz zwischen der durch Transhumanz und Holzwirtschaft geprägten Bergwirtschaft zur Landwirtschaft.

67 Hild / Hellenkemper (1986) 54, 60, 66-68, 92; TIB 5,1 260 s.v. 'Gökburç'; CJ VIII 10, 10; CIL XII 1524; vgl. Demandt (1989) 335; SCHUler (1998) 84.

68 HopwOOD (1999a) 194.

69 Repertorium 373, 'Sel 137' = BM II 197, Nr. 218; SCHULER (1998) 276.

70 Amm. XXVII 9, 7; s.u.

71 Strab. XII 6, 5; vgl. Mitchell (1995a) 71. 
Miracula an. ${ }^{72}$ Doch hier zeigt sich kein konkreter Hinweis für diese These, vielmehr gibt es in den Miracula zahlreiche Stellen, mit denen man das Gegenteil belegen kann, so den Fall des Sohnes der isaurischen Geisel Bassiane, der es in Eirenopolis zu Ansehen gebracht hatte. ${ }^{73}$ Angehörige der isaurischen Oberschicht, die nach Konfliktfällen der römischen Obrigkeit Geiseln stellte, waren in isaurischen Städten angesehene Bürger. Somit sind es nicht „die“ Isaurier als Volk, welche der Autor der Miracula anklagt, sondern vielmehr die isaurischen Räuber. Die Isaurier werden in den Miracula deshalb niemals als solche benannt, sondern immer mit mit den Ausdrücken „Räuber" oder „Plagegeister" umschrieben.

Ein generelles Wohlstandsgefälle zwischen Stadt und Land, oder gar das Bild von zwei ,getrennten Welten“, kann für das spätantike Kleinasien daher nicht angenommen werden. Beide Bereiche, der ländliche und der städtische Raum, ergänzten sich vielmehr gegenseitig; bis zu einem gewissen Grade bestand sogar wechselscitige Abhängigkeit, da die Poleis von den auf ihrer Chora gewonnen Produkten abhingen. ${ }^{74}$ Erst im Zuge der Krisen des 3. und 4. Jahrhunderts kam es zu Nahrungsmittelknappheiten, welche, dies sei vorweggenommen, eine der Hauptursachen für die isaurischen Aufstände darstellten. ${ }^{75}$ Ammians Beschreibung Isauriens als fruchtbares Land (palmita viret et frugibus multis) traf zwar wohl hauptsächlich auf die Ebene im Binnenland und den Küstenstreifen $\mathrm{zu}^{76}$ dennoch ist in den Quellen von vielen landwirtschaftlichen Produkten auch des Hinterlandes die Rede. Eine kleinasiatische Spezialität war das Styrax (auch liquidamber orientalis genannt), ein aromatisches, klebriges Gummiharz, das als Produkt eines Wurmparasiten auf dem gleichnamigen Baum gewonnen wurde. Es wurde gesammelt und entweder pur verwendet oder mit dem Blütenstaub des Baumes vermischt. Frauen benützten es, um ihr Haar damit zu parfümieren. ${ }^{77}$ Außer in Syrien kam es auch in Isaurien vor: Ein kilikischer Freund des Redners Libanius namens Acacius ließ diesem regelmäßig Styrax aus dem isaurischen Hinterland zukommen. ${ }^{78}$

72 Hellenkemper (1986) 627 unter Verweis auf Mir. 28; vgl. Dagron (1978) 122: im isaurischen Brigantentum zeige sich ,l'antique opposition entre les cités et les tribus de l'intérieur".

73 Mir. 19; vgl. Dagron (1978) 118 u. III.2.2.3.

74 Vgl. BRANDT (1992) $132 \mathrm{f}$.

75 S.u. IV.3.2.3.

76 Amm. XIV 8, 1; vgl. HoPWOOD (1989a) 192

77 Strab. XII 7, 3; in Selge: XVI 4, 13. 26; Plin. n.h. I 12a. XXIV 24f,; Ciris 168; Baum: Hdt. III 107; Plut. Lys. 28; Andrew DALBY, Empire of Pleasures. Luxury and indulgence in the Roman world, London 2000, 167, 170.

78 Lib. ep. 338; PLRE I 6f. s.n. 'Acacius 7'. 1139, Stemma 15. 
Wolle aus Kilikien und Isaurien wurde in die Städte und von dort ins ganze Reich verkauft; die Kilikier galten als besonders tüchtige Schafscherer. ${ }^{79}$ Die Bergwälder des Taurus waren so ertragreich, daß Zedernholz für den Schiffsbau exportiert wurde. ${ }^{80}$ Noch im 5. und 6. Jahrhundert wurden Ziegenhaar und Schafwolle zu Schiffstauen verarbeitet. Isaurien war durch die Schiffahrt in den Wirtschaftskreislauf des römischen Reiches eingebunden. ${ }^{81}$

Auf welche Weise außerhalb der Polisgrenzen die Rechtsordnung und der Friede aufrecht erhalten wurden, darüber läßt sich allenfalls spekulieren. ${ }^{82}$ Es kann als wahrscheinlich angenommen werden, daß die Landsitze der Ratsleute in den Chorai ihrer Poleis als Basen für die Verteilung von Schutzgarantien an die Landbewohner fungierten. Durch diese Tätigkeit könnten die Bouleuten die Loyalität ihrer ländlichen Klientel gewonnen haben. ${ }^{83}$ Inwieweit und mit welcher zeitlichen Dauer sich diese Einflußzonen quer über das Land auch auf entfernt liegende Gruppen erstreckten, läßt sich jedoch angesichts der sehr mageren Quellenlage nicht entscheiden. Auch darüber, wie die Isaurier zu großen Gruppen zusammenfanden, wie deren soziale Zusammensetzung war und vor allem: ob und welche Ziele diese Gruppen verfolgten, geben die Quellen bestenfalls indirekte Auskünfte. ${ }^{84}$ Die einzige einen derartigen Sachverhalt andeutende Stelle findet sich bei Ammian. Hier handeln die Einwohner von Germanicopolis, der größten Stadt in der isaurischen Dekapolis, aufgrund ihres Ansehens bei der Landbevölkerung einen Frieden zwischen den römischen Truppen und den aufständischen Isauriern des Hinterlandes aus. Ammian gibt somit einen deutlichen Hinweis auf enge Beziehungen zwischen den Gruppierungen in den Städten und in deren Hinterland. ${ }^{85}$

79 Mart. XIV 140; Columella Praef. 26; Plin. n.h. VIII 201; Varro rust.II 11; Plin. n.h. VIII 201; Suet. Aug. 75; Strab. XII 6, 1; vgl. Ferdinand ORTH, s. v. 'Lana', RE XII 1 (1924) 606f.; HopwOOD (1991) 307.

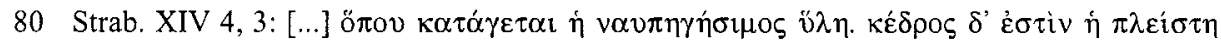
[...]; vgl. HeLLENKEMPER (1986) 626.

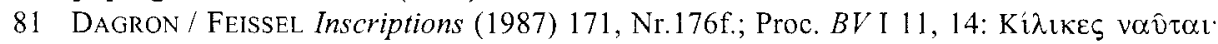
vgl. TIB 5,1 117. 141 zu Gewerbe, Handwerk und Schiffahrt.

82 Hierzu s. auch u. Kap. III.3.1.2.

83 So Hopwood (1986) passim. (1999a) 194.

84 Hierzu MatTHEws (1989) 359: „Village settlements connected to each other by social and economic ties now hidden, but important in their day suggesting the possibility that in Isauria also the communities might have formed groups capable, like the Homonadenses, of concerted action."

85 Amm. XXVII 9, 7. Hopwood (1986) 350f. u. (1999b) 231f. zur Vermittlertätigkeit der Einwohner von Germanicopolis; Hinweis auf wirtschaftliche Interessen und soziale Verflechtungen zwischen Polisbewohnern und Rebellen bei MATTHEws (1989) 365; auch ToMASCHITZ (1998) sieht in Ammians quorum apud eos ut signiferea manus semper valuere sententiae ein engeres Verhältnis als die bloße Vermittler-Rolle. - Zu den Hintergründen des Aufstands s.u. Kap. IV.2,1.1. 
Die oben genannten frühen Quellen sprechen von den großen Erträgen von Schafwolle und Ziegenhaar aus Kilikien und damit auch Isaurien, was die Existenz von größeren Herden voraussetzt. Diese konnten in der bergigen Gegend nur durch Transhumanz unterhalten werden, die komplexe Praxis der Fernweidewirtschaft. ${ }^{86}$ Eine Reihe von Pfaden aus Küstenstädten ins Innenland läßt die Transhumanz in der Frühzeit vermuten. ${ }^{87}$ Die in Städten oder Ortschaften fest ansässige Bevölkerung ließ dabei ihren Bestand an Kleinviehherden durch Lohnhirten oder Angehörige auf die oftmals weit entfernt liegenden Weidegründe treiben.

In der Forschung wird nun immer wieder ein Fließgleichgewicht zwischen dem Hirten- und dem Banditenstatus der Aufständischen behauptet: Jeder Hirte sei bei Bedarf zum Banditen geworden. Bei der Entstehung des Banditentums in der antiken Gesellschaft überhaupt komme dem Phänomen der Transhumanz eine tragende Rolle zu. ${ }^{88}$ Die immer in der freien Natur tätigen Schäfer fielen, wohl oftmals nicht zu Unrecht, unter den Generalverdacht des Banditentums. Es waren wohl selbst die kaiserlichen Schafhirten im Nebenberuf Räuber gewesen, denn im Jahr 409 verbot der Westkaiser Honorius, sie als Zieheltern zuzulassen, und belegte sie im entsprechenden Gesetz mit dem Begriff societas latronum. Um ihren Aktionsradius einzuschränken, wurde ihnen der Gebrauch von Pferden untersagt. ${ }^{89}$ Von ,Sklavenhirten“ im Sinne der Latifundien des 1. Jahrhunderts v. Chr. (und entsprechenden sozialgeschichtlichen Implikationen) kann angesichts der veränderten Gesellschaftsstruktur für das spätantike Isaurien jedoch nicht gesprochen werden, und es gibt keine Hinweise, daß die isaurischen Hirten in der Mehrzahl Sklaven gewesen sind. ${ }^{90}$ Aus der Transhumanz, wenn sie denn im spätantiken Isaurien noch eine Rolle gespielt hat, auf einen inneren Gegensatz zwischen städtischem und ländlichem Raum zu schließen, ist aus mehreren Gründen problematisch:

86 Gerhard WaldherR, s.v. 'Transhumanz' in: SonNABEND (1999), 564-568 definiert diese als „globales Phänomen“, ,gekennzeichnet durch eine klimabedingte, saisonale periodische und alternierende Wanderung von größeren Kleinviehherden“ und als „Zwischenstufe auf dem Weg vom Vollnomadismus zur Seßhaftigkeit".

87 HopwoOd (1989a) 192-195; Russell (1991) 487, Anm. 118.

88 So SHAW (1984) 31.

89 CTh IX 30, 2. 5 (Pferde). 31 (Zieheltern); s. auch CJ IX 2, 11 ,id est pastorum latronum$v e^{\text {ck; }}$ Fronto ep. ad Marcum Caesarem II 12; nach SHAw (1984) 31 waren die Hirten auch die treibende Kraft hinter den Sklavenaufständen, dagegen weist HoPwOOD (1999a) 180 darauf hin, daß die Hirten allein niemals zahlreich genug für einen größeren Aufstand gewesen seien; es mußten immer noch Bauern hinzukommen. Dies sei der Grund, warum die meisten Banditen-Rebellionen mehr in Eric HoBSBAwMs Typus des „Rächers“ passen als in jenes des ,social bandit“, HobsBawm ( $\left.{ }^{2} 1972\right)$; vgl. auch Demandt (1989) 335; RiEss (2001) $58 \mathrm{f}$.

90 So jedoch RIEss (2001) $58 \mathrm{f}$. 
Die Rolle der Transhumanz war insgesamt von weitaus geringerer Bedeutung als von HOPWOOD angenommen. ${ }^{91}$ Der Großteil der Quellen zeigt, auch wenn die Transhumanz nirgends explizit belegt ist, daß die Isaurier seit der Kaiserzeit größtenteils in Städten lebten und Ackerbau und Viehzucht betrieben. Dieser Umstand bildet einen Widerspruch zu der hier dargelegten Transhumanz-Praxis, und bereits während der Hohen Kaiserzeit war diese Form der Weidewirtschaft nicht mehr die vorherrschende Produktionsweise in der Region. ${ }^{92}$ Bereits zu dieser frühen Zeit ist von gegenseitigen Überfällen unter Hirten zum Zweck des Viehraubs und der Gleichung „Bandit = Räuber" keine Rede in den Quellen, von der Spätantike ganz zu schweigen.

Die von der Transhumanz und einem damit verbundenen Beziehungsgeflecht zwischen weit im Inland gelegenen Punkten und der Küste ausgehende Annahme HOPWOODs, daß selbst die in den Küstenstädten ansässigen einflußreichen Bürger über Einfluß und Ländereien im isaurischen Hochland verfügten, ${ }^{93}$ ist ebenfalls nirgendwo belegt. Das einzige Zeugnis, das er anführt, stammt aus der hellenistischen Zeit. Hierbei handelt es sich um eine Inschrift auf einem unter Ptolemaios gebauten Serapistempel, der im 2. Jahrhundert n. Chr. erneuert wurde. Sie deutet auf einen Disput zwischen den Städten Olosada und Thouththurbia (Odunçu Kalesı bei Ha$\mathrm{d} ı \mathrm{~m})^{94}$ hin, zwei oberhalb des zerklüfteten Kalykadnostals an der antiken Straße von Iconium nach Germanicopolis gelegenen Orten. Der Disput wurde durch die zwei Tagesreisen östlich liegende, mächtigere Polis Colybrassus geschlichtet. Nach der (in diesem zeitlichen Bezug) plausiblen Interpretation Hopwoods hatte sich dieser Streit um Weidegründe entzündet. Colybrassus habe damit gleichzeitig seine Interessen vertreten, da es seine Hirten auf das Territorium der beiden Städte geschickt habe. ${ }^{95}$ Doch dieses Einzelzeugnis für städtische Einflußnahme auf weit auseinander liegende Weidegründe kann nicht als Basis für eine generelle Gültigkeit 600 Jahre später postuliert werden. Die hohe Relevanz, welche dieser Form der Weidewirtschaft von SHAW und HOPWOOD für den Charakters des isaurischen Widerstands gegen die römische Herrschaft beigemessen wird, ist nicht zuletzt angesichts der überaus dürftigen Quellenlage als gering anzusehen. Für die in der Spätantike auftretenden und sich über den östlichen Mittelmeerraum ausweitenden Konflikte zwischen Isauriern und Römern kann der Transhumanz kein bestimmender Einfluß zugeschrieben werden, denn diese Konflikte gingen aufgrund ihres hohen Organisationsgrades über die Di-

91 HOPWOOD (1989a) 192-195 verweist mangels schriftlicher Quellen auf eine für Italien geltende Studie: Joan M. FRAYN, Sheep rearing and the wool trade in Roman Italy (ARCA 15), Liverpool 1984.

92 So LENSKI (1999a) 447f.

93 HopwOOD (1991). (1999a).

94 BM II 139f., Nr. 138 (Olosada), 141-144, Nr. 139-144 (Thuththurbia); TIB 5,1 370 s.v. 'Olosada', 445 s.v. 'Thuththurbia'; HoPwood (1989a) 195f.: ,admittedly in the Hellenistic period“". Die Orte sind $7 \mathrm{~km}$ nördlich von Taşkent gelegen.

95 Nach BM II 140 wurde Colybrassus durch seine Serapis-Priester vertreten. 
mension einfacher Hirten-Revolten weit hinaus. ${ }^{96}$ Isaurische Mächtige strebten dauerhafte Positionen an, namentlich die beiden Zenones und Illus im 5. Jahrhundert stehen am Ende dieser Entwicklung. ${ }^{97}$

Insgesamt ist eine vielfältige Wechselbeziehung zwischen Stadt und Land zu konstatieren. Damit ist die von Autoren wie Strabo und Plinius postulierte gesetzmäßige Dichotomie zwischen hellenisierter bzw. romanisierter Küstenlandschaft und bergigem, barbarisch gebliebenen Hinterland, wie sie für Isaurien am pointiertesten durch SHAW übernommen wurde, nicht gerechtfertigt. ${ }^{98}$ Die gegenseitige Abhängigkeit der Hirten als Produzenten von den städtischen Märkten und der Städter von den Hirten als Konsumenten kann als Beleg für eine weit fortgeschrittene Romanisierung gelten. ${ }^{99}$ Der von den antiken Autoren behauptete und von der Forschung teilweise wiederholte Gegensatz von Stadt und Land bzw. Ebene und Berg läßt sich nicht einmal für die folgende Zeit des 3. und 4. Jhs. aufrecht erhalten. Daraus ergibt sich eine gegenüber dem Großteil der bisherigen Forschung differenzierte Deutungsgrundlage für die Ereignisse der nun folgenden Jahrhunderte.

96 So LeNSKi (1999a) 417, 447f. gegen die von SHAw (1990) vertretene These von der Transhumanz als konstitutivem Element eines isaurischen Dauer-Widerstandes. SHAW (1990) geht noch von einer zumindest halbnomadischen Weidewirtschaft aus, während LENSKI l.c. auf die Urbanisierung Isauriens verweist; HoPwoon (1999a) 195 zur Erklärung von (angenommenen) Konflikten unter isaurischen Hirten. Er nimmt die Existenz von zwei Gruppen an, einer aus dem Binnenland und einer von der - seiner Ansicht nach - höher urbanisierten Küste, welche sich in gegenseitiger Abhängigkeit befunden hätten.

97 S.u. Kap. V.2.

98 Zum gleichen Ergebnis für Afrika kommt Alan Rushworth, 'North African deserts and mountains: comparisons and insights' in: David L. KENNEDY (Hrsg.), The Roman Army in the East (= JRA Suppl. series 18), Ann Arbor, Mich. 1996, 297-316, gegen die frühere Forschung, die eine stetige résistance vermutet hatte.

$99 \mathrm{Zu}$ diesem Ergebnis kommt auch BRANDT (1992) in seinem Vergleich zwischen dem bergigen Pisidien und dem ebenen, urbanisierten Pamphylien. Zur Abhängigkeit der Hirten von den städtischen Märkten vgl. HoPwOOD (1986) 349. 